\title{
Application Based Production Management System In Textile Industries
}

\author{
Yoganathan. N. S a, Sangeetha. $S^{b}$, Nithya Devi. $N^{c}$, Abishek. $M^{d}$, Sangeetha. $E^{e}$ \\ $\mathbf{a , b , c , d , \mathbf { e }}$ Department of ECE, Sona College of Technology, Salem, India
}

Article History: Received: 10 November 2020; Revised 12 January 2021 Accepted: 27 January 2021; Published online: 5 April 2021

\begin{abstract}
Presently, there is lack of proper management system to manage orders and analyze the daily reports which is prerequisite to acquire production management systems. This paper objectives to elucidate the production management system in small and medium sized Enterprises manufacturing apparent in India. SMES rely invariably on register and manual - data sheet based management which are detrimental in the management process. This proposed system provides a cost-effective solution to the above said problem through app-based platform, it reveals the production planning through reports of the data collected to analyze the daily production on various parameters involved in apparel production. This system provides a daily report to the authorized person (Owner/ Supervisor) to facilitate production management and solve issues pertaining to production output addressing various implements involved in the process.
\end{abstract}

Keyword: Android app- Hardware - Real time monitoring - Order management

\section{Introduction}

Man is a chum of style in nature. The higher preference for better garment and apparel resulted in the development of textile fiber manufacturing and textile manufacturing process. Production planning is a complicated area of any production operation [1]. Textile production planning is complex as there exists various types of fibers, yarn counts, spinning systems processing methods and end products, all of the factors combined with the computer's requirement correctly filled orders and quick delivery on time, make the production planning system more complex.

In addition, effective production planning in textile industry has grown to be increasingly crucial as excessive foreign opposition has impacted the market[2]. Textile production systems form an fascinating area for the study of scheduling difficulties. We are planning to present a very good model for production planning and measurement and thereby provide complete satisfaction to the customers.

\section{Literature Review}

Real time management of data was done in many ways. Everett Zraket and Benington in 1957 advanced a real time data management procedure for the air defense system[3]. In real-time management, the crucial elements were the gathering of information, quick processing, brief answer and spontaneous feedback .

The automatic identification methods and capture information (AIDC) is answerable for the data gathering, automatically and in real time using 2 main ideologies: gathering data through radiofrequency and sensors. The Radio Frequency Identification (RFID) is not an undiscovered technology and was initially used for defense functions during World War II [4]. RFID has various advantages when applied in a manufacturing system by Brewer, Sloan \& Landers in 1999, from identification to distance, regular screening products, real-time management and their functioning in intense conditions .

Sensory gadgets are the most common factors in control systems in real time as they gather data on a large number of parameters[5]. According to their integration, when synchronized with the strategic plan of the company, permits reaching increased productivity, quality, reliability and time reduction. In 2007, Tanenbaum \& Van Steen mentioned the major goals of a distributed system: resource sharing, openness, concurrency, scalability, fault tolerance and transparency[6]. 
Over the above mentioned reviews, managing the production on a handy mobile phone will be a sophisticated solution for the owners to make their industry the best in the market.

\section{Existing Methodology}

The existing method of production management uses paper and record based management of production. This method of production management will increase the need for man power. The boon of Technology is to reduce man power[7]. To reduce man power, we need a better solution. The Solution for this should be provided by the blooming engineers. The man power can increase the expenditure of owner. It can also cause human errors[8]. When a staff in this designation is absent, then the process of management requires someone else to complete.

The calculation of order's deadline is also based on manual process and may cause errors at sometimes. Calculating deadlines in small scale industries is very rare. Also, in large Scale industries, man power is used for it. Calculating deadlines and fixing target is must for an efficient management [9]. This helps in the growth of an Industry. Expecting a man to do everything will not be permanent forever. Instead of seeking for labors. What if we get our own predictions of target. There is where our future mobile world is. Industrialists are in need of such Technologies [10][11].

Calculating the target for each day is not accurate if done manually. Most of the industries lag in growth because they lack in firing target [12]. Fixing the target for each day and even for each hour improves the management in industries. Now a days, Most of the industries is not firing their target[13] [17]. This is resulting in the lowered efficiency of industry. Even though they fix the target, they may be less accurate resulting in missing deadline and thereby getting bad opinion in the market.

\section{Proposed Methodology}

Our project is developed on the basis of real time order and production management in textile industries. We designed an android application that can run in android devices having versions greater than 4.0. Our model is too satisfactory in bringing the needs of a customer and the owner's management.

\subsection{Block Diagram}

The smart, app-based production management system has both hardware and software parts. When the power is on, pic value is read using manual input [14]. Each rotation of shaft represents one wrap of cloth the rotation is monitored using proximity sensor [15][16]. For each rotation count increments. After an hour, the production value is measured using

\section{production $=$ count $/$ pic}

The above value I in inches. This value is sent to database via a Wi-Fi module from which a mobile app retrieve.

The following Figure 1 represents the schematic diagram of the proposed application-based production management system in textile industries.

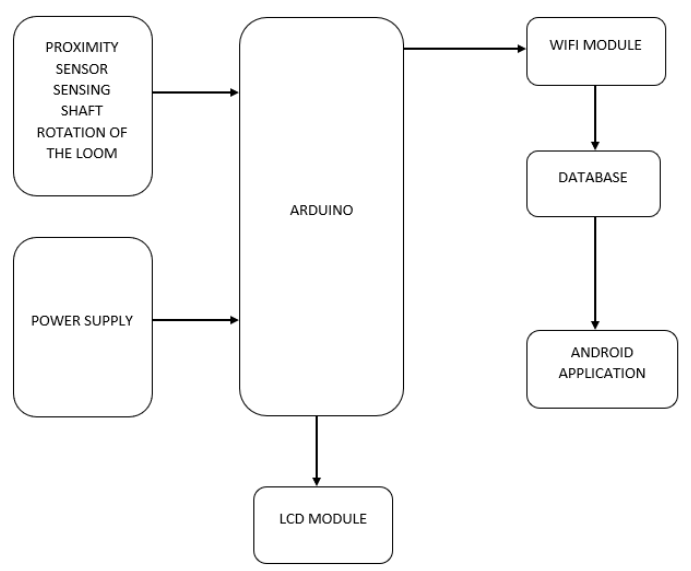

Figure 1. Schematic diagram of the proposed application-based production management system. 


\subsection{Flow Chart}

The flow chart of our proposed model is depicted in Figure 2. It involves the following steps:

Step 01:

Step 02:

Step 03:

Step 04:

Step 05:

Step 06:

Step 07:

Step 08:

Step 09:

Step 10:

start.

read the pic value.

set count $\mathrm{c}=0$.

if one rotation of shaft is completed, go to step 5 .

increment c by 1 .

if time is one hour, go to step 7, else go to step 4.

calculate production value in inches. Production $=\mathrm{c} / \mathrm{pic}$.

upload this value to database.

Step 11:

retrieve this value from database using remote mobile application.

if the system is switched off go to step 11, else go to step 3.

stop.

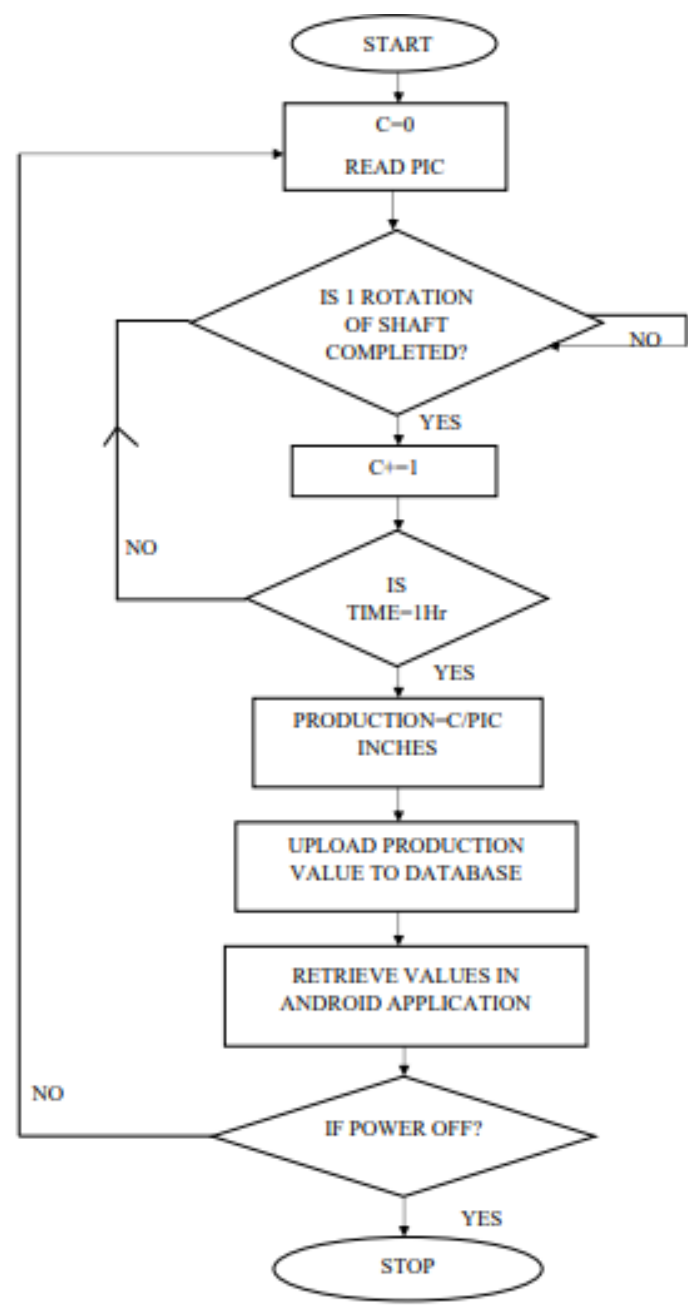

Figure 2. Flow Chart

\section{Results}

The production management system management maintains orders of various customers and maintains a record of information for each and every order. Also, the daily production details are also recorded for each customer's order.

The Figure 3 depicts the order taking procedure in the application we designed. In the main page of our application, we placed a button for taking the order. On clicking this button, we will be directed to the page for calculating the production deadline. Upon filling the details and clicking the button, we will be displayed the 
time required to achieve this production and the daily and hourly target calculated by the application itself. Upon entering the name of order and clicking the button below that, we will be directed to the main page itself in which the new order will be there.

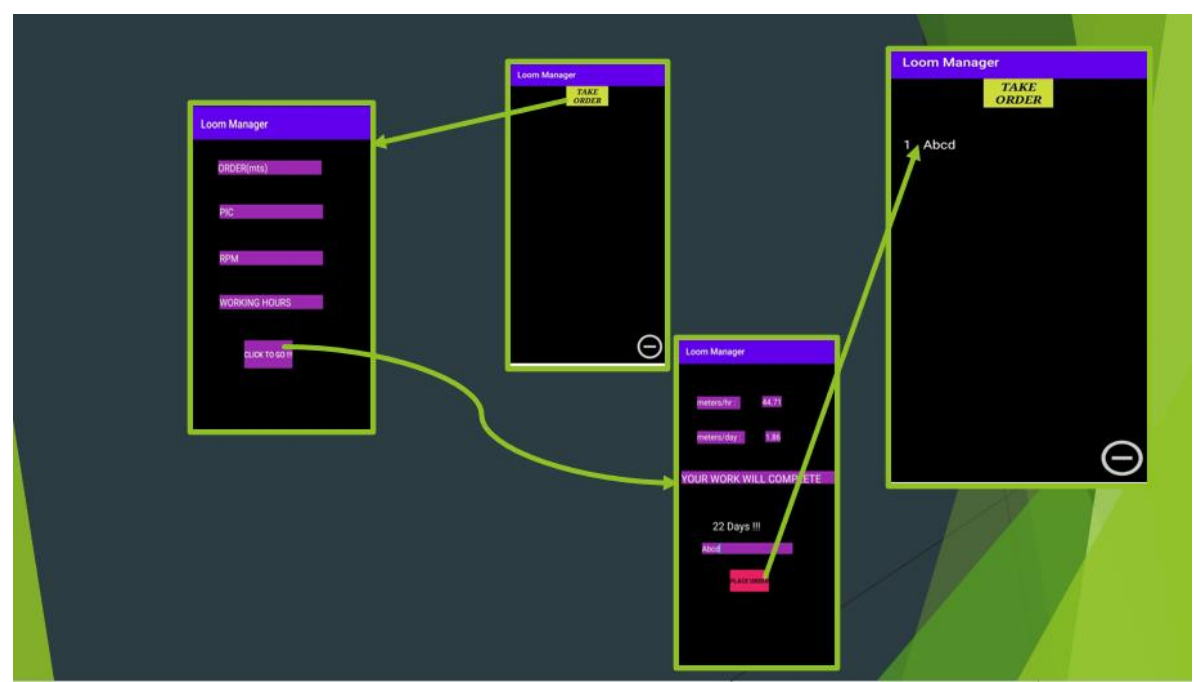

Figure 3. Application View 1

The Figure 4 depicts the inner functions in each order. In the main page, we have to click on the order name in order to check its details. On clicking the specified order, we will be directed to a page with 2 tabs one providing the details of order and the other providing the daily report of the production value fetched from the database.

If we want to delete an order, we have to click on the - symbol in the main page and select the order to be deleted and click on the delete button.

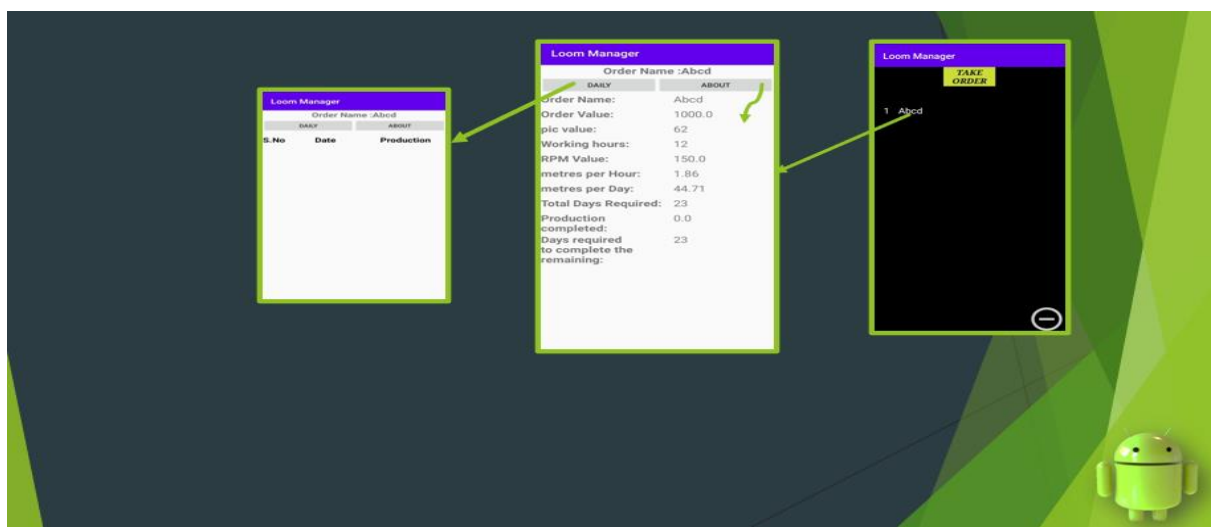

Figure 4. Application View 2

\section{Conclusion}

This paper was described as a proposed platform for supporting a wide range of industrial management functions through integrated modules which includes data processing and communication with different kinds of machines and tools.

This system will provide a great goodness to the weavers across the world and it will be a great monitoring process for the growing textile industries thus making them a grown industry in the future.

\section{REFERENCES}

1. Alexandru, C. \& Gheorghe, G. (2015). 'Production Management Software', International Conference on Numerical Analysis and Applied Mathematics, Vol. 1648, Article number 620003.

2. Brewer, A., Sloan, N., \& Landers, T. L. (1999). 'Intelligent tracking in manufacturing', Journal of Intelligent Manufacturing, Vol. 10, No. 3, pp. 245- 250. 
3. Everett, R. R., Zraket, C., \& Benington, H. (1957). 'SAGE: A dataprocessing system for air defense.'. The Romanian Review Precision Mechanics, Optics \& Mechatronics, 2016, Issue 50127.

4. G. Adamopoulos, N. Karacapilidis, S. Pantazopoulos, (1994) 'Production Management In The Textile Industry Using The "Yfadi" Decision Support System”, Dept. of Mechanical Engineering, University of Patras, 26500 Rio, Patras, Greece

5. Thiyaneswaran, B., Anguraj, K., Kumarganesh, S., \& Thangaraj, K. (2020). Early detection of melanoma images using gray level co-occurrence matrix features and machine learning techniques for effective clinical diagnosis. International Journal of Imaging Systems and Technology, (ima.22514). doi:10.1002/ima.22514

6. G. G. Vieira, M. L. R. Varela, G. D. Putnik, J. M. Machado, J. Trojanowska (2016), 'Integrated Platform for Real-time Control and Production and Productivity Monitoring and Analysis', The Romanian Review Precision Mechanics, Optics \& Mechatronics, 2016, Issue 50.

7. Sandhiya, D., \& Thiyaneswaran, B. (2017). Extraction of dorsal palm basilic and cephalic hand vein features for human authentication system. 2017 International Conference on Wireless Communications, Signal Processing and Networking (WiSPNET). IEEE.

8. Jagannathan, Jayanthi. "Current Trends: Machine Learning and AI in IoT." Integrating the Internet of Things Into Software Engineering Practices (2019): 181-198.

9. Gunasekaran, A., Ngai, E. W., \& McGaughey, R. E. (2006), 'Information technology and systems justification: A review for research and applications', European Journal of Operational Research, 173(3), pp. 957-983.

10. Haynes, M., McGregor, A., \& Stewart, N. (1997), ' The business team standard: A means of improving the effectiveness of individual businesses in a multibusiness corporation.', Systems practice, Vol. 10, No.3,pp. 219239.

11. B Thiyaneswaran, K Anguraj, M Sindhu, N. S Yoganathan, J Jayanthi. (2020). Development of Iris Biological Features Extraction for Biometric Based Authentication to Prevent Covid Spread. International Journal of Advanced Science and Technology, 29(3), 8266-8275.

12. Hedler Carvalho, Ana Rocha, Joao . Monteiro Luis F. Silva(2003), 'Planning, Control and Monitoring Tools for Industrial Garment Assembly Processes' ICIT 2003 - Maribor, Slovenia.

13. Leandro L. Lorente Leyva, Edwin P. Curillo Perugachi, Ramiro V. Saraguro Piarpuezan, Carlos A. Machado Orges and Edwin P. Ortega Montenegro (2018 ),'Lean Manufacturing Application in Textile Industry', Proceedings of the International Conference on Industrial Engineering and Operations Management Paris, France, July 26-27, 2018.

14. Jayanthi, J., Lydia, E. L., Krishnaraj, N., Jayasankar, T., Babu, R. L., \& Suji, R. A. (2020). An effective deep learning features based integrated framework for iris detection and recognition. Journal of Ambient Intelligence and Humanized Computing, 1-11.

15. B Thiyaneswaran, V Bhuvaneshwaran, M.S Dharun, K Gopu, T Gowsikan (Ed.). (2020). Breathing level monitoring and alerting by using embedded IOT (Vol. 10). Journal of Green Engineering.

16. López, T. S., Ranasinghe, D. C., Patkai, B., \& McFarlane, D. (2011). 'Taxonomy, technology and applications of smart objects', Information Systems Frontiers, Vol. 13, No. 2, pp. 281-300.

17. Monostori, L., Csáji, B. C., Kádár, B., Pfeiffer, A., Ilie-Zudor, E., Kemény, Z., \& Szathmári, M. (2010), 'Towards adaptive and digital manufacturing', Annual Reviews in Control, Vol. 34, No. 1, pp. 118-128.

18. Nikos I. Karacapilidis, Costas P. Pappis (1996), 'Production planning and control in textile industry: A case study', Department of industrial Management, University of Piraeus, Greece.

19. Parmenter, D. (2010). 'Key performance indicators (KPI): developing, implementing, and using winning KPIs: John Wiley \& Sons'.

20. Thiyaneswaran, B., Saravanakumar, A., \& Kandiban, R. (2016). Extraction of mole from eye sclera using object area detection algorithm. 2016 International Conference on Wireless Communications, Signal Processing and Networking (WiSPNET). IEEE.

21. Soloman, S. (2009). 'Sensors and control systems in manufacturing (2 ed.): McGraw-Hill, Inc'.

22. Suhail Anwar, Priyesh Deep(2017), 'App based production monitoring system for apparel small and medium sized industries', Amity journal operations management. 